\section{Honeybees and 'yellow rain'}

SIR-The misidentification as a biological warfare agent of the 'yellow rain' produced by defaecation during massed flights of the giant honeybee, Apis dorsata, in South-East Asia', has focused attention on the phenomenon of mass defaecation. We now suggest it can be explained by the need to prevent the temperature of bees' nests becoming so hot as to endanger the larvae.

When honeybees remain close together in the hot and humid tropics, their corporately generated heat must be dissipated. European honeybees ( $A$. mellifera) evaporate water to keep the hive's temperature below about $37^{\circ} \mathrm{C}$, the thermal limit for larval life $\mathrm{e}^{2,3}$. Individuals of $A$. mellifera flying in extreme heat regurgitate nectar, let it evaporate on their mouthparts, and re-ingest the cooled droplet $^{4}$ (a cycle we call gobbetting). Apis dorsata does the same both in the colony and in flight, maintaining a brood-rearing temperature of about $33.5^{\circ} \mathrm{C}$ (ref. 5).

In tropical forests of Asia, $A$. dorsata nests in colonies of about 40,000 bees on large, single combs suspended in the open from high, breezy supports ${ }^{6,7}$. Some aspects of their thermoregulatory behaviour are well known ${ }^{5,8}$. At $26^{\circ} \mathrm{C}$, the midpoint of the daily ambient temperature, the temperature of the thorax in individual bees in a colony is at least $4.6^{\circ} \mathrm{C}$ above that of the ambient air. The temperature excess $\left(T_{\mathrm{E}}\right)$ of the thorax at ambient temperatures above or below $26^{\circ} \mathrm{C}$ is more than $4.6^{\circ} \mathrm{C}$, a result of thermoregulatory activity for cooling and warming, respectively.

One striking behaviour of $A$. dorsata is mass flight from the broodright colony, in which at least half of a colony's bees fly in gentle arcs up to about $20 \mathrm{~m}$ from the comb 5-6 min before returning. We have observed 19 such flights. In each case, the flying bees defaecated, a behaviour resembling cleansing flights in $A$. mellifera. We found $65-100 \%$ of 306 bees in six colonies had faeces before mass flight, but only $0-44 \%$ did so afterwards. The average volume of faeces dumped was $18.3 \pm$ $3.2 \mu \mathrm{l}$, weighing $24.6 \pm 2.8 \mathrm{mg}$ or $20 \%$ of the total body weight. The mass specific heat of the faeces was $1.85 \mathrm{cal}$ per $\mathrm{g}{ }^{\circ} \mathrm{C}$. With the minimum abdominal $T_{\mathrm{E}}$ of relatively inactive curtain bees of $2.52 \pm$ $0.99^{\circ} \mathrm{C}$, a typical colony would dump 1,850 cal during mass defaecatory flights at ambient temperatures between 28 and $31{ }^{\circ} \mathrm{C}$ (ref. 5).

Apart from physical loss of heat in mass flight, reduction of a bee's body mass greatly increases thermoregulatory efficiency. Given a heat coefficient of $0.83 \mathrm{cal}$ per $\mathrm{g}{ }^{\circ} \mathrm{C}$ for animal tissue, the amount of energy needed to be lost to reduce the $T_{\mathrm{E}}$ of $5.1 \pm 1.1{ }^{\circ} \mathrm{C}$ before mass flight to $3.4 \pm$ $1.0^{\circ} \mathrm{C}$ afterwards would be $0.22 \mathrm{cal}$ for a bee which has not defaecated (mean weight $123 \mathrm{mg}$ ), but only $0.14 \mathrm{cal}$ for one which had (mean weight $98 \mathrm{mg}$ ). That is an energy saving of $36 \%$ per participating bee and at least $15 \%$ for the colony. The brood's $T_{\mathrm{E}}$ before and after mass flight was not significantly different.

Mass flight and defaecation occur when thermal stress is greatest. Of recorded mass flights, $60 \%$ occurred between 0900 and 1200 when temperatures rise most rapidly, and another $20 \%$ between 1600 and 1800 , when it is often windless. For 33 flights where wind was recorded, 29 occurred in windless periods. The average ambient temperature during 17 mass flights was $29.5 \pm 1.0^{\circ} \mathrm{C}$, with a relative humidity of $53 \pm 6 \%$. Gobbetting always occurred before and after mass flights, indicating the continuing need for cooling. The number of gobbetting bees increases as the ambient temperature rises, whether or not mass flight occurs, and gobbetting bees always have lower $T_{\mathrm{E}}$ than do nongobbetting ones ${ }^{5}$.

At air temperatures above $29^{\circ} \mathrm{C}$ the bees' $T_{\mathrm{E}}$ rises to and above $36^{\circ} \mathrm{C}$ (ref. 5). Under still, humid conditions when heat is not easily lost to the air, temperatures in the curtain and brood rise above toleration (about $\left.37^{\circ} \mathrm{C}\right)^{\mathrm{s}}$. Thus, activities which increase efficiency of heat loss, such as mass defaecation, can help avoid the nest temperature reaching a lethal temperature (about $37^{\circ} \mathrm{C}$ ) under still humid conditions when heat is not easily lost to the air and when the ambient temperature is high.

\section{Plant Protection Department,} Universitiy Pertanian Malaysia,

Serdang, Selangor, Malaysia 43400

PETER G. KeVAn

Department of Environmental Biology,

University of Guelph,

Guelph, Ontario, Canada N1G 2W1

\title{
Ceramics and flocculation
}

SIR-Kendall et al. ${ }^{\prime}$ convincingly demonstrate the importance of particle clusters in limiting the strength of ceramics, but their analysis of the clustering process is incorrect.

The application of nucleation concepts to particle aggregation is open to serious doubt. The external surface energy of a cluster of particles must be less than the total surface energy of the individual particles that make up the cluster, because of particle-particle contacts. This effect would favour cluster formation rather than oppose it, as implied by equation (2) of Kendall et al. ${ }^{\text {. }}$. In nucleation from solution, the formation of new crystal surface causes an increase in surface energy, which is outweighed by cohesive energy only for nuclei larger than a certain critical size.

Even if the analysis leading to their equation (4) is accepted as correct for clusters, the stationary value is not a dominant cluster size, but a critical size corresponding to a maximum of free energy. Clusters smaller than this size would be unstable, whereas for larger clusters, continued growth is favoured; there should be no dominant size. In practice, shear conditions will impose an upper limit on cluster size, but this aspect does not enter the treatment of Kendall et al.

With this conceptual error removed, there is no longer the apparent paradox that weaker attraction leads to larger aggregates, nor do the speculations con- cerning biological growth have any foundation. The common-sense view that strong inter-particle attraction leads to large aggregates is still essentially correct. JOHN GREGORY

Department of Civil Engineering, University College London,

Gower Street,

London WC1E 6BT, UK

Kendall REPLIES-We did not claim a formal analogy between nucleation theory and growth of particle clusters. There cannot be a formal analogy because particle aggregates can exhibit a large range of packing and structure whereas crystals are generally of uniform structure.

Our experiments, however, showed that flocculating a suspension of fine particles causes agglomerates to grow, somewhat like crystal growth, whereas vigorous agitation of the suspensions causes the aggregates to break down, unlike crystal growth. Therefore it seemed reasonable to conceive of a balance of growth against breakdown such that clusters of a particular size were produced under the influence of brownian motion.

Such a balance was obtained by considering the free energy of an agglomerate. The outer surface has a positive free energy and so tends to shrink, whereas the contact points within the agglomerate have a negative free energy and so tend to promote growth. The balance between these processes was found by seeking the stationary value of the sum of these posi- 